\title{
Bone mineral density in adults with osteoarthritis or rheumatoid arthritis: a cross-sectional study of a nationwide population.
}

\section{Zhongxin Zhu}

Zhejiang Chinese Medical University

Gangfeng Hu

Hangzhou Xiaoshan No 1 People's Hospital

Hongting Jin

Zhejiang Chinese Medical University

Peijian Tong ( $\square$ tongpeijian@163.com )

Zhejiang Chinese Medical University https://orcid.org/0000-0003-0948-8674

Research article

Keywords: osteoarthritis; degenerative arthritis; rheumatoid arthritis; bone health; NHANES

Posted Date: May 15th, 2020

DOI: https://doi.org/10.21203/rs.3.rs-28236/v1

License: (c) (i) This work is licensed under a Creative Commons Attribution 4.0 International License.

Read Full License 


\section{Abstract \\ Background}

It is reported that osteoporosis commonly occurs in rheumatoid arthritis (RA), whereas there is a controversial relationship with osteoarthritis (OA). In this study, we aimed to investigate the relations between $O A, R A$ and bone mineral density (BMD) in adults aged 20-59 years.

\section{Methods}

We merged and analyzed the database from the National Health and Nutrition Examination Survey (NHANES) 2011-2018. Arthritis status and types of arthritis were obtained from questionnaires. Lumbar BMD was measured by dual-energy $X$-ray absorptiometry. The associations between OA, RA and lumbar BMD were evaluated using logistic regression models. Subgroup analyses stratified by gender and race were performed. The association between disease duration of arthritis and lumbar BMD was also investigated.

\section{Results}

A total of 11094 adults aged 20-59 years were analyzed in this study. Compared with the non-arthritis group, participants with OA had a higher lumbar BMD ( $\beta=0.023 ; 95 \% \mathrm{Cl}: 0.011-0.035)$, while no significant association was observed in rheumatoid arthritis $(\beta=0.014 ; 95 \% \mathrm{Cl}:-0.003-0.031)$. In the subgroup analyses stratified by gender, males with $O A$ had a higher lumbar BMD compared with those without ( $\beta=0.014 ; 95 \% \mathrm{Cl}:-0.003-0.031)$. However, this association changed in females $(\beta=0.007 ; 95 \%$ $\mathrm{Cl}$ - $0.008-0.021)$. On the other hand, no significant association was observed in RA in both males and females (males: $\beta=0.023 ; 95 \% \mathrm{Cl}:-0.003-0.048$, females: $\beta=0.008 ; 95 \% \mathrm{Cl}:-0.015-0.031$ ). Moreover, the disease duration of arthritis was not associated with lumbar BMD in both OA $(\beta=-0.0001 ; 95 \% \mathrm{Cl}$ : $-0.0017-0.0015)$ and RA ( $\beta=0.0006 ; 95 \% \mathrm{Cl}:-0.0012-0.0025)$.

\section{Conclusions}

The correlation between OA and lumbar BMD differed by sex. Patients with OA were more likely to have higher lumbar BMD in males, but not in females. On the other hand, no significant association was found in RA both in males and females.

\section{Introduction}

Osteoporosis (OP), osteoarthritis (OA), and rheumatoid arthritis (RA) are a series of disorders called musculoskeletal pathologies that cause intolerable pains, movement disorders, and even permanent disability[1]. With the aging of the social population, these diseases have reached about 1 in 4 adults in 
developed countries[2, 3]. RA is an autoimmune disease of unknown etiology with a homeostatic imbalance[4]. As a biomechanical disease, the onset and development of OA are closely related to inflammatory and catabolic alterations[5]. OP is a systemic disease associated with a dramatic loss of bone mineral density (BMD). As two notable silent rheumatic diseases, OA and OP have been included on the World Health Organization disease-disabling list $[1,6]$.

It is reported that OP commonly occurs in RA, whereas there is a controversial relationship with OA[7-10]. In this study, we therefore investigated the relations between OA, RA and BMD in adults aged 20-59 years using a population-based sample from the National Health and Nutrition Examination Survey (NHANES) database.

\section{Methods}

\section{Study population}

The NHANES program is a series of surveys focusing on health topics of US population of all ages. The data collection and analysis require special statistical analysis due to the multistage, complex clustered probability design of the survey, rather than based on a simple random sample of the US population[11].

The current study used the data from NHANES 2011-2018. The study population was restricted to adults aged 20-59 years (enrollment from NHANES 2011-2018, $n=14934$ ). Participants without data on arthritis diagnosis $(n=23)$, lumbar BMD $(n=3398)$, or with cancer $(n=419)$ were excluded. Finally, a total of 11094 subjects were analyzed in this study. The ethics review board of the National Center for Health Statistics (NCHS) approved all protocols, and written informed consent was obtained from each participant[12]

\section{Arthritis}

A diagnosis of arthritis was based on the medical conditions questionnaires collected by NHANES during the household interviews. Arthritis was classified as OA, RA, psoriatic arthritis, other, and don't know (or refused) if the participants reported ever being told they had arthritis by a doctor or other health professional. We defined the disease duration of arthritis as age in years at screening minus age when the doctor told the participants they had arthritis.

\section{Lumbar BMD}

Lumbar spine is one site typically evaluated for the assessment and treatment of osteoporosis, and BMD measurement at this site has been used as a clinical trial outcome for over three decades[13]. As the most widely accepted method, the dual-energy X-ray absorptiometry (DXA) scans provide bone measurements for lumbar spine. In NHANES program, the scans were acquired on the Hologic Discovery model A densitometers (Hologic, Inc., Bedford, Massachusetts), and were analyzed with Hologic APEX version 4.0 software. Further details of the DXA examination protocol are located on the NHANES website. 


\section{Demographic data and other questionnaire data}

Age, gender, race, educational level, ratio of family income to poverty, vigorous recreational activities, and smoked at least 100 cigarettes in life were recorded using questionnaires managed by intervieweradministers. Race was self-reported as Non-Hispanic White, Non-Hispanic Black, Mexican American, Other Hispanic, and Other race-including multi-racial. The description of vigorous recreational activities was based on the patient self-report described as the following question: 'Do you do any vigorousintensity sports, fitness, or recreational activities that cause large increases in breathing or heart rate like running or basketball for at least 10 minutes continuously?'

\section{Laboratory data}

Biospecimens were collected for laboratory analysis to provide detailed information on the nutritional status and health of the participants. The collecting, processing, storing, and shipping of the biospecimens took place in the mobile examination center. The detailed measurement processes of blood urea nitrogen, total protein, total cholesterol, alkaline phosphatase, serum potassium, serum sodium, serum phosphorus, serum uric acid, and serum calcium can be found at the NHANES website.

\section{Statistical analysis}

To assure national representation, we used weighted analysis recommended by the analytical guideline edited by NCHS. P-value was calculated using a weighted chi-square test for categorical variables and using a weighted linear regression model for continuous variables.

The relations between arthritis and lumbar BMD were examined using multivariable logistic regression. Three models were constructed: model 1, no covariates were adjusted; model 2, age, gender, race were adjusted; model 3, age, gender, race, educational level, body mass index, ratio of family income to poverty, vigorous recreational activities, smoked at least 100 cigarettes in life, blood urea nitrogen, total protein, total cholesterol, alkaline phosphatase, serum potassium, serum sodium, serum phosphorus, serum uric acid, and serum calcium were adjusted. Besides, following the STROBE guideline[14], we performed subgroup analyses to make better use of the data. We also performed multivariable logistic regression to explore the association between disease duration of arthritis and lumbar BMD. We performed all analyses using package R version 3.4.3 (http://www.R-project.org) and EmpowerStats software (http://www.empowerstats.com). The significance level was set to 0.05 .

\section{Results}

\section{Study sample}

The characteristics of the samples are presented in Table 1. The mean age of the participants with arthritis (47.99 \pm 9.36 years) was significantly higher than the mean age of those without arthritis (37.59 \pm 11.34 years). Compared with the non-arthritis group, arthritis group had a higher proportion of females (56.08\% vs. $46.17 \%)$. Race, educational level, body mass index, vigorous recreational activities, smoked at 
least 100 cigarettes in life, blood urea nitrogen, total protein, total cholesterol, alkaline phosphatase, serum uric acid, serum sodium, and serum calcium were also significantly different between the two groups $(p<0.05)$.

\section{Multiple regression model}

As shown in Table 2, the association between arthritis and lumbar BMD was not significant in the unadjusted model $(\beta=0.007 ; 95 \% \mathrm{Cl}:-0.001-0.015)$. However, this association became significant after adjustment (model 2: $\beta=0.017 ; 95 \% \mathrm{Cl}$ : 0.008-0.025, model 3: $\beta=0.018 ; 95 \% \mathrm{Cl}: 0.010-0.027$ ). Moreover, we evaluate the relationships between different types of arthritis and lumbar BMD. Compared with the non- arthritis group, participants with osteoarthritis or degenerative arthritis and psoriatic arthritis had a higher lumbar BMD (osteoarthritis or degenerative arthritis: $\beta=0.023 ; 95 \% \mathrm{Cl}: 0.011-0.035$, psoriatic arthritis: $\beta=0.024 ; 95 \% \mathrm{Cl}: 0.005-0.043$ ), while no significant association was observed in RA $(\beta=0.014 ; 95 \% \mathrm{Cl}:-0.003-0.031)$.

\section{Subgroup analyses}

In the subgroup analyses stratified by gender (Table 3), male adults with osteoarthritis or degenerative arthritis had a higher lumbar BMD compared with those without ( $\beta=0.014 ; 95 \% \mathrm{Cl}:-0.003-0.031$ ). However, this association changed in female adults $(\beta=0.007 ; 95 \% \mathrm{Cl}:-0.008-0.021)$. On the other hand, no significant association was observed in RA both in males and females (males: $\beta=0.023 ; 95 \% \mathrm{Cl}$ : $-0.003-0.048$, females: $\beta=0.008 ; 95 \% \mathrm{Cl}:-0.015-0.031$ ).

In the subgroup analyses stratified by race (Table 4), non-Hispanic White adults with osteoarthritis or degenerative arthritis had a higher lumbar BMD compared with those without $(\beta=0.014 ; 95 \% \mathrm{Cl}$ : $-0.003-$ 0.031). no significant associations were observed in other osteoarthritis or degenerative arthritis groups or any RA groups.

\section{Associations between disease duration of arthritis and lumbar BMD.}

As shown in Table 5, the disease duration of arthritis was not associated with lumbar BMD both in osteoarthritis or degenerative arthritis $(\beta=-0.0001 ; 95 \% \mathrm{Cl}:-0.0017-0.0015)$ and $\mathrm{RA}(\beta=0.0006 ; 95 \% \mathrm{Cl}$ : $-0.0012-0.0025)$.

\section{Discussion}

The present study demonstrated that patients with OA were more likely to have higher lumbar BMD in males, but not in females. On the other hand, no significant association was found in RA in both males and females. 
Despite years of research, the association of OP with OA is still discussed. Both diseases depend on bone metabolism and positively correlated with aging. In a study comprised 359 postmenopausal women aged 50-89 years, Povoroznyuk et al[15] found that women with a symptomatic OA had a significantly higher lumbar BMD compared with controls. The cross-sectional data of a Korean national survey found a negative association of lumbar BMD with the presence of knee OA[16]. A recent prospective study provided strong evidence that high femoral neck BMD is a prognostic risk factor for the development of knee and hip radiographic $O A[17]$. In addition, higher BMD has been shown to reduce the risk of fractures in both men and women[18, 19]. The concomitant presence of OP and OA in patients with hip or spine OA were also reported[20, 21].

The biologic mechanism by which BMD influences OA has not been established, and previous statistically significant findings may result from uncontrolled and unmeasured confounding factors, such as skeletal growth factors[22], bone geometry[23, 24], bone morphology[25], and genetic[26]. In this study, we found the correlation between $\mathrm{OA}$ and lumbar BMD differed by sex. One possible explanation is that high $\mathrm{BMI}$ and weight-bearing activities, which increase the risk of damage to articular cartilage leading to $\mathrm{OA}$, are also beneficial to the preservation of bone mass.

OP in RA patients may be mediated through several mechanisms: pro-inflammatory state, glucocorticoids use, low level of physical activity, and the classic risk factors for OP[27]. However, in a cross-sectional study including 152 Korean, Kweon et al[28] found no significant difference in lumbar BMD between patients with RA aged over 50 years and control individuals. In a study of 138 postmenopausal RA patients, Mori et al[29] found that disease duration was significantly related to BMD using multivariate linear regression analyses. On the contrary, in a study of 76 RA patients, the results suggested that the reduction in $\mathrm{BMD}$ was less than expected in the first decade of the disease compared with the reference population[30]. In a study of 299 Korean female patients with RA, Lee et al[31] found no significant association between disease duration of RA and BMD. Our data suggested there were no significant associations between RA, disease duration of RA and BMD in both males and females. The reasons for the different conclusions may be attributed to the variations among these studies, including demographic characteristics, sample size, study design, and controlled confounders.

The strengths of this study include a population-based sample with a wide age range that is generalizable to a community population, subgroup analyses for sensitivity test, and adjustment for many potential confounders. There are also several limitations. First, due to the cross-sectional nature of this study, we were unable to elucidate the causal relationship between arthritis and BMD. More longitudinal studies investigating the causality between them are needed. Second, the diagnosis of arthritis was based on the patient's self-report that might be subject to bias. However, the consistency of self-reported arthritis and clinical confirmation has been documented[32,33]. Third, the missing information on different sites of arthritis preclude us to estimate the associations between OA, RA and BMD in specific sites. Fourth, study results cannot be generalized to the participants with cancer because these special populations were excluded in the analyses. Finally, there might be other confounding factors we did not control for in our study. For example, glucocorticoids used for the treatment of RA were 
not adjusted in this study. However, it was reported that there was no significant association between cumulative glucocorticoid dose and BMD after adjustment of confounders [31]. The results of a population-based study also showed no significant difference between corticosteroid treated patients with RA and non-steroid group, indicating that the independent effect of corticosteroids on BMD is only minimal[34].

In summary, our results indicated that there is an inverse relationship between OP and the presence of OA in males, but not in females. There was no significant association between RA and lumbar BMD in both males and females. Our findings may provide some important implications for better understanding the linking between OA, RA and bone health.

\section{Declarations}

\section{Author contributions}

ZXZ, HTJ contributed to data collection, analysis and writing of the manuscript. PJT contributed to study design and writing of the manuscript.

\section{Funding}

This study received no funding.

\section{Consent for publication}

Not applicable.

\section{Competing interests}

The authors declare that they have no competing interests.

\section{Ethical Statement}

The ethics review board of the National Center for Health Statistics approved all NHANES protocols and written informed consents were obtained from all participants.

\section{Acknowledgements}

The authors appreciate the time and effort given by participants during the data collection phase of the NHANES project.

\section{References}

1. Franco-Trepat E, Guillan-Fresco M, Alonso-Perez A, Jorge-Mora A, Francisco V, Gualillo O, Gomez R: Visfatin Connection: Present and Future in Osteoarthritis and Osteoporosis. Journal of clinical 
medicine $2019,8(8)$.

2. Barbour KE, Helmick CG, Boring M, Brady TJ: Vital Signs: Prevalence of Doctor-Diagnosed Arthritis and Arthritis-Attributable Activity Limitation - United States, 2013-2015. MMWR Morbidity and mortality weekly report 2017, 66(9):246-253.

3. National Collaborating Centre for Chronic C: National Institute for Health and Clinical Excellence: Guidance. In: Rheumatoid Arthritis: National Clinical Guideline for Management and Treatment in Adults. London: Royal College of Physicians (UK) Royal College of Physicians of London.; 2009.

4. Mclnnes IB, Schett G: The pathogenesis of rheumatoid arthritis. The New England journal of medicine 2011, 365(23):2205-2219.

5. Gomez R, Villalvilla A, Largo R, Gualillo O, Herrero-Beaumont G: TLR4 signalling in osteoarthritisfinding targets for candidate DMOADs. Nature reviews Rheumatology 2015, 11(3):159-170.

6. Geusens PP, van den Bergh JP: Osteoporosis and osteoarthritis: shared mechanisms and epidemiology. Current opinion in rheumatology 2016, 28(2):97-103.

7. Clayton ES, Hochberg MC: Osteoporosis and osteoarthritis, rheumatoid arthritis and spondylarthropathies. Current osteoporosis reports 2013, 11(4):257-262.

8. Ramonda R, Sartori L, Ortolan A, Frallonardo P, Lorenzin M, Punzi L, Musacchio E: The controversial relationship between osteoarthritis and osteoporosis: an update on hand subtypes. International journal of rheumatic diseases 2016, 19(10):954-960.

9. Im GI, Kim MK: The relationship between osteoarthritis and osteoporosis. Journal of bone and mineral metabolism 2014, 32(2):101-109.

10. Dequeker J, Aerssens J, Luyten FP: Osteoarthritis and osteoporosis: clinical and research evidence of inverse relationship. Aging clinical and experimental research 2003, 15(5):426-439.

11. Dillon CF, Weisman MH: US National Health and Nutrition Examination Survey Arthritis Initiatives, Methodologies and Data. Rheumatic diseases clinics of North America 2018, 44(2):215-265.

12. Zipf G, Chiappa M, Porter KS, Ostchega Y, Lewis BG, Dostal J: National health and nutrition examination survey: plan and operations, 1999-2010. Vital and health statistics Ser 1, Programs and collection procedures 2013(56):1-37.

13. Kanis JA, Johnell O: Requirements for DXA for the management of osteoporosis in Europe. Osteoporosis international : a journal established as result of cooperation between the European Foundation for Osteoporosis and the National Osteoporosis Foundation of the USA 2005, 16(3):229238.

14. von Elm E, Altman DG, Egger M, Pocock SJ, Gotzsche PC, Vandenbroucke JP: The Strengthening the Reporting of Observational Studies in Epidemiology (STROBE) statement: guidelines for reporting observational studies. Lancet (London, England) 2007, 370(9596):1453-1457.

15. Povoroznyuk VV, Zaverukha NV, Musiienko AS: Bone mineral density and trabecular bone score in postmenopausal women with knee osteoarthritis and obesity. Wiadomosci lekarskie (Warsaw, Poland : 1960) 2020, 73(3):529-533. 
16. Kim YH, Lee JS, Park JH: Association between bone mineral density and knee osteoarthritis in Koreans: the Fourth and Fifth Korea National Health and Nutrition Examination Surveys. Osteoarthritis and cartilage 2018, 26(11):1511-1517.

17. Bergink AP, Rivadeneira F, Bierma-Zeinstra SM, Zillikens MC, Ikram MA, Uitterlinden AG, van Meurs JBJ: Are Bone Mineral Density and Fractures Related to the Incidence and Progression of Radiographic Osteoarthritis of the Knee, Hip, and Hand in Elderly Men and Women? The Rotterdam Study. Arthritis \& rheumatology (Hoboken, NJ) 2019, 71(3):361-369.

18. Stone KL, Seeley DG, Lui LY, Cauley JA, Ensrud K, Browner WS, Nevitt MC, Cummings SR: BMD at multiple sites and risk of fracture of multiple types: long-term results from the Study of Osteoporotic Fractures. Journal of bone and mineral research : the official journal of the American Society for Bone and Mineral Research 2003, 18(11):1947-1954.

19. Cummings SR, Cawthon PM, Ensrud KE, Cauley JA, Fink HA, Orwoll ES: BMD and risk of hip and nonvertebral fractures in older men: a prospective study and comparison with older women. Journal of bone and mineral research : the official journal of the American Society for Bone and Mineral Research 2006, 21(10):1550-1556.

20. Chan MY, Center JR, Eisman JA, Nguyen TV: Bone mineral density and association of osteoarthritis with fracture risk. Osteoarthritis and cartilage 2014, 22(9):1251-1258.

21. Lingard EA, Mitchell SY, Francis RM, Rawlings D, Peaston R, Birrell FN, McCaskie AW: The prevalence of osteoporosis in patients with severe hip and knee osteoarthritis awaiting joint arthroplasty. Age and ageing 2010, 39(2):234-239.

22. Hunter DJ, Spector TD: The role of bone metabolism in osteoarthritis. Current rheumatology reports 2003, 5(1):15-19.

23. Ding C, Cicuttini F, Jones G: Tibial subchondral bone size and knee cartilage defects: relevance to knee osteoarthritis. Osteoarthritis and cartilage 2007, 15(5):479-486.

24. Javaid MK, Lane NE, Mackey DC, Lui LY, Arden NK, Beck TJ, Hochberg MC, Nevitt MC: Changes in proximal femoral mineral geometry precede the onset of radiographic hip osteoarthritis: The study of osteoporotic fractures. Arthritis and rheumatism 2009, 60(7):2028-2036.

25. Nelson AE, Golightly YM, Renner JB, Schwartz TA, Liu F, Lynch JA, Gregory JS, Aspden RM, Lane NE, Jordan JM: Variations in Hip Shape Are Associated with Radiographic Knee Osteoarthritis: Crosssectional and Longitudinal Analyses of the Johnston County Osteoarthritis Project. J Rheumatol 2016, 43(2):405-410.

26. Yau MS, Yerges-Armstrong LM, Liu Y, Lewis CE, Duggan DJ, Renner JB, Torner J, Felson DT, McCulloch CE, Kwoh CK et al: Genome-Wide Association Study of Radiographic Knee Osteoarthritis in North American Caucasians. Arthritis \& rheumatology (Hoboken, NJ) 2017, 69(2):343-351.

27. Ibanez M, Ortiz AM, Castrejon I, Garcia-Vadillo JA, Carvajal I, Castaneda S, Gonzalez-Alvaro I: A rational use of glucocorticoids in patients with early arthritis has a minimal impact on bone mass. Arthritis research \& therapy 2010, 12(2):R50. 
28. Kweon SM, Sohn DH, Park JH, Koh JH, Park EK, Lee HN, Kim K, Kim Y, Kim GT, Lee SG: Male patients with rheumatoid arthritis have an increased risk of osteoporosis: Frequency and risk factors. Medicine (Baltimore) 2018, 97(24): e11122.

29. Mori Y, Kuwahara Y, Chiba S, Kogre A, Baba K, Kamimura M, Itoi E: Bone mineral density of postmenopausal women with rheumatoid arthritis depends on disease duration regardless of treatment. Journal of bone and mineral metabolism 2017, 35(1):52-57.

30. Kroot EJ, Nieuwenhuizen MG, de Waal Malefijt MC, van Riel PL, Pasker-de Jong PC, Laan RF: Change in bone mineral density in patients with rheumatoid arthritis during the first decade of the disease. Arthritis and rheumatism 2001, 44(6):1254-1260.

31. Lee SG, Park YE, Park SH, Kim TK, Choi HJ, Lee SJ, Kim SI, Lee SH, Kim GT, Lee JW et al: Increased frequency of osteoporosis and BMD below the expected range for age among South Korean women with rheumatoid arthritis. International journal of rheumatic diseases 2012, 15(3):289-296.

32. El Miedany Y, El Gaafary M, Youssef SS, Palmer D: Incorporating patient reported outcome measures in clinical practice: development and validation of a questionnaire for inflammatory arthritis. Clinical and experimental rheumatology 2010, 28(5):734-744.

33. March LM, Schwarz JM, Carfrae BH, Bagge E: Clinical validation of self-reported osteoarthritis. Osteoarthritis and cartilage 1998, 6(2):87-93.

34. Kroger $\mathrm{H}$, Honkanen $\mathrm{R}$, Saarikoski S, Alhava E: Decreased axial bone mineral density in perimenopausal women with rheumatoid arthritis-a population based study. Annals of the rheumatic diseases 1994, 53(1):18-23.

\section{Tables}

Table 1 Characteristic of study sample with and without arthritis. 


\begin{tabular}{|c|c|c|c|}
\hline & Arthritis $(\mathrm{n}=1510)$ & Non-arthritis(n=9584) & $\mathrm{P}$ value \\
\hline Age (years) & $47.99 \pm 9.36$ & $37.59 \pm 11.34$ & $<0.0001$ \\
\hline Age groups & & & $<0.0001$ \\
\hline 20-29 years & 5.97 & 30.31 & \\
\hline 30-39 years & 12.38 & 26.12 & \\
\hline 40-49 years & 27.09 & 24.53 & \\
\hline $50-59$ years & 54.56 & 19.04 & \\
\hline Gender $(\%)$ & & & $<0.0001$ \\
\hline Male & 43.92 & 53.83 & \\
\hline Female & 56.08 & 46.17 & \\
\hline Race (\%) & & & $<0.0001$ \\
\hline Mexican American & 6.07 & 11.06 & \\
\hline Other Hispanic & 5.07 & 7.71 & \\
\hline Non-Hispanic White & 68.41 & 58.80 & \\
\hline Non-Hispanic Black & 12.47 & 12.32 & \\
\hline Other race - including multi-racial & 7.97 & 10.11 & \\
\hline Educational level (\%) & & & $<0.0001$ \\
\hline Less than 9th grade & 3.94 & 3.96 & \\
\hline 9-11th grade & 11.83 & 9.02 & \\
\hline High school graduate/GED or equivalent & 22.99 & 21.67 & \\
\hline Some college or AA degree & 35.13 & 32.47 & \\
\hline College graduate or above & 26.11 & 32.87 & \\
\hline Not recorded & 0 & 0.01 & \\
\hline Body mass index $(\mathrm{kg} / \mathrm{m} 2)$ & $31.71 \pm 8.00$ & $28.56 \pm 6.56$ & $<0.0001$ \\
\hline Ratio of family income to poverty & $2.95 \pm 1.72$ & $2.94 \pm 1.66$ & 0.8213 \\
\hline Vigorous recreational activities (\%) & & & $<0.0001$ \\
\hline Yes & 19.99 & 36.63 & \\
\hline No & 80.01 & 63.37 & \\
\hline Smoked at least 100 cigarettes in life (\%) & & & $<0.0001$ \\
\hline Yes & 51.38 & 38.63 & \\
\hline No & 48.59 & 61.35 & \\
\hline Not recorded & 0.03 & 0.02 & \\
\hline Blood urea nitrogen $((\mathrm{mmol} / \mathrm{L})$ & $4.82 \pm 1.71$ & $4.54 \pm 1.49$ & $<0.0001$ \\
\hline Total protein $(\mathrm{g} / \mathrm{L})$ & $70.60 \pm 4.45$ & $71.58 \pm 4.29$ & $<0.0001$ \\
\hline Total cholesterol $((\mathrm{mmol} / \mathrm{L})$ & $5.11 \pm 1.04$ & $4.91 \pm 1.02$ & $<0.0001$ \\
\hline Alkaline phosphatase (U/L) & $71.50 \pm 22.57$ & $66.32 \pm 22.73$ & $<0.0001$ \\
\hline Serum uric acid(umol/L) & $323.79 \pm 84.78$ & $318.53 \pm 80.72$ & 0.0191 \\
\hline Serum sodium $(\mathrm{mmol} / \mathrm{L})$ & $139.12 \pm 2.43$ & $139.25 \pm 2.21$ & 0.0369 \\
\hline Serum potassium $(\mathrm{mmol} / \mathrm{L})$ & $3.95 \pm 0.33$ & $3.97 \pm 0.31$ & 0.1215 \\
\hline Serum phosphorus (mmol/L) & $1.20 \pm 0.17$ & $1.20 \pm 0.18$ & 0.3971 \\
\hline Serum calcium (mmol/L) & $2.34 \pm 0.09$ & $2.34 \pm 0.08$ & 0.0129 \\
\hline Disease duration of arthritis (years) & $9.20 \pm 8.90$ & 1 & \\
\hline Which type of arthritis was it? (\%) & & & 1 \\
\hline Osteoarthritis or degenerative arthritis & 41.61 & 1 & \\
\hline Rheumatoid arthritis & 17.45 & 1 & \\
\hline Psoriatic arthritis & 2.73 & 1 & \\
\hline Other & 0.17 & 1 & \\
\hline Don't know or refused & 24.20 & 1 & \\
\hline Lumbar bone mineral density $\left(\mathrm{g} / \mathrm{cm}^{2}\right)$ & $1.05 \pm 0.16$ & $1.04 \pm 0.15$ & 0.0861 \\
\hline
\end{tabular}

Mean \pm SD for continuous variables: P value was calculated by weighted linear regression model.

\% for categorical variables: P value was calculated by weighted chi-square test.

Table 2 Associations between arthritis and lumbar bone mineral density. 


\begin{tabular}{lccc}
\hline & Model 1 & Model 2 & Model 3 \\
& $\beta(95 \%$ CI, P) & $\beta(95 \%$ CI, P) & $\beta(95 \%$ CI, P) \\
\hline Non- Arthritis & Reference & Reference & Reference \\
Arthritis & $0.007(-0.001,0.015) 0.0861$ & $0.017(0.008,0.025) 0.0001$ & $0.018(0.010,0.027)<0.0001$ \\
\hline Non- Arthritis & Reference & Reference & Reference \\
Osteoarthritis or degenerative arthritis & $0.013(0.001,0.025) 0.0293$ & $0.023(0.012,0.035) 0.0001$ & $0.023(0.011,0.035) 0.0001$ \\
Rheumatoid arthritis & $0.000(-0.017,0.018) 0.9733$ & $0.010(-0.007,0.027) 0.2599$ & $0.014(-0.003,0.031) 0.1074$ \\
Psoriatic arthritis & $0.005(-0.039,0.049) 0.8177$ & $0.020(0.000,0.039) 0.0445$ & $0.024(0.005,0.043) 0.0133$ \\
Don't know or refused & $0.000(-0.015,0.015) 0.9712$ & $0.008(-0.006,0.023) 0.2665$ & $0.011(-0.004,0.026) 0.1350$ \\
\hline
\end{tabular}

Model 1: no covariates were adjusted.

Model 2: age, gender, race were adjusted.

Model 3: age, gender, race, educational level, body mass index, ratio of family income to poverty, vigorous recreational activities, smoked at least 100 cigarettes in life, blood urea nitrogen, total protein, total cholesterol, alkaline phosphatase, serum uric acid, serum sodium, serum potassium, serum phosphorus, and serum calcium were adjusted.

Table 3 Subgroup analyses stratified by gender.

\begin{tabular}{|c|c|c|c|}
\hline & $\begin{array}{c}\text { Model } 1 \\
\beta(95 \% \text { CI, P) } \\
\end{array}$ & $\begin{array}{c}\text { Model } 2 \\
\beta(95 \% \text { CI, P) } \\
\end{array}$ & $\begin{array}{c}\text { Model 3 } \\
\beta(95 \% \text { CI, P) } \\
\end{array}$ \\
\hline Non- Arthritis & Reference & Reference & Reference \\
\hline Rheumatoid arthritis & $0.010(-0.016,0.036) 0.4569$ & $0.021(-0.005,0.046) 0.1139$ & $0.023(-0.003,0.048) 0.0776$ \\
\hline \multicolumn{4}{|l|}{ Female } \\
\hline Non- Arthritis & Reference & Reference & Reference \\
\hline
\end{tabular}

Associations were adjusted for age, race, educational level, body mass index, ratio of family income to poverty, vigorous recreational activities, smoked at least 100 cigarettes in life, blood urea nitrogen, total protein, total cholesterol, alkaline phosphatase, serum uric acid, serum sodium, serum potassium, serum phosphorus, and serum calcium.

Table 4 Subgroup analyses stratified by race.

\begin{tabular}{lccc}
\hline & Model 1 & Model 2 & Model 3 \\
& $\beta(95 \%$ CI, P) & $\beta(95 \%$ CI, P) & $\beta(95 \%$ CI, P) \\
\hline Non-Hispanic White & Reference & Reference & Reference \\
Non- Arthritis & $0.009(-0.009,0.026) 0.3439$ & $0.018(-0.000,0.036) 0.0501$ & $0.019(0.001,0.037) 0.0382$ \\
Osteoarthritis or degenerative arthritis & & & \\
Rheumatoid arthritis & $-0.015(-0.045,0.015) 0.3222$ & $-0.007(-0.036,0.023) 0.6699$ & $0.001(-0.028,0.031) 0.9232$ \\
Non-Hispanic Black & Reference & Reference & Reference \\
Non- Arthritis & $-0.020(-0.050,0.009) 0.1806$ & $0.003(-0.027,0.034) 0.8340$ & $0.007(-0.024,0.037) 0.6611$ \\
Osteoarthritis or degenerative arthritis & $0.008(-0.031,0.046) 0.6947$ & $0.032(-0.007,0.071) 0.1105$ & $0.032(-0.007,0.071) 0.1035$ \\
Rheumatoid arthritis & & & \\
Mexican American & Reference & Reference & Reference \\
Non- Arthritis & $0.015(-0.025,0.056) 0.4579$ & $0.032(-0.008,0.073) 0.1189$ & $0.017(-0.023,0.057) 0.4103$ \\
Osteoarthritis or degenerative arthritis & $-0.022(-0.061,0.018) 0.2899$ & $-0.004(-0.044,0.036) 0.8602$ & $-0.006(-0.046,0.033) 0.7621$ \\
Rheumatoid arthritis & &
\end{tabular}

Associations were adjusted for age, gender, educational level, body mass index, ratio of family income to poverty, vigorous recreational activities, smoked at least 100 cigarettes in life, blood urea nitrogen, total protein, total cholesterol, alkaline phosphatase, serum uric acid, serum sodium, serum potassium, serum phosphorus, and serum calcium. 
Table 5 Associations between disease duration of arthritis and lumbar bone mineral density.

\begin{tabular}{lccc}
\hline Disease duration of arthritis (years) & Model 1 & Model 2 & Model 3 \\
& $\beta(95 \%$ CI) P value & $\beta(95 \%$ CI) P value & $\beta(95 \%$ CI) P value \\
\hline Osteoarthritis or degenerative arthritis & $0.0005(-0.0021,0.0011) 0.5312$ & $-0.0003(-0.0020,0.0013) 0.6746$ & $-0.0001(-0.0017,0.0015) 0.8619$ \\
& $0.0013(-0.0005,0.0032) 0.1596$ & $0.0013(-0.0005,0.0032) 0.1649$ & $0.0006(-0.0012,0.0025) 0.4979$ \\
\hline
\end{tabular}

Associations were adjusted for age, race, educational level, body mass index, ratio of family income to poverty, vigorous recreational activities, smoked at least 100 cigarettes in life, blood urea nitrogen, total protein, total cholesterol, alkaline phosphatase, serum uric acid, serum sodium, serum potassium, serum phosphorus, and serum calcium. 